\title{
A NEW MODEL FOR THE DETERMINATION OF POINT DEFECT EQUILIBRIUM CONCENTRATIONS IN SILICON
}

\author{
M. Budil, E. Guerrero, T. Brabec, S. Selberherr, H. Poetzl \\ Department of Physical Electronics, Institute for General Electrical Engineering and Electronics, \\ Technical University Vienna and Ludwig Boltzmann-Institute for Solid State Physics, \\ Vienna, Austria.
}

\section{ABSTRACT}

In this paper the boundary conditions for point defect distributions in monocrystalline silicon are investigated. These boundary conditions are established by simple thermodynamic considerations. A circle process is used including vacancy, interstitial and Frenkel pair generation which yields a simple relationship between the vacancy and interstitial equilibrium concentrations at the surface. A new OED model is also presented which explains the $t^{-1 / 4}$ behaviour of the interstitial supersaturation. This model is used to simulate experiments of Mizuo and Higuchi. In this way values for the equilibrium concentrations and the diffusion coefficients of vacancies and interstitials are obtained.

\section{Introduction}

For a precise prediction of dopant profiles in silicon the understanding of the influence of thermal oxidation on the diffusion of dopants is of great importance. It is well established that oxidation enhanced diffusion (OED) and oxidation retarded diffusion (ORD) result from the change of the distribution of vacancies (V) and interstitials (I) by the thermal oxidation process ([1-3] and references therein). In order to clarify OED/ ORD a new model is presented for the interaction between the oxidized interface and the point defects. The most essential feature of this model is the influence of oxidation on the equilibrium point defect concentrations which are not taken as constant bulk parameters but as resulting from the chemical processes at the interfaces of the semiconductor crystal. Chapter 2 introduces this basic concept which is applied to oxidizing conditions in chapter 3 . In chapter 4 a process simulator [4] is applied to determine the physical parameters of the new model from experimental results available in literatur [5-8].

2. The thermal equilibrium of point defects

In this chapter we shall derive a general law for the equilibrium concentration of point defects. This law ensues from a circle process using the Frenkel mechanism. First some definitions are given: A point defect is a local disorder of the crystal lattice. There exist three different ways to generate point defects.

-An atom is removed from his lattice place and is brought to a surface or an interface position. Simultanously there remains an empty site (V). This kind of point defect is called Schottky defect. The Gibbs potential to produce such a defect is $\Delta G_{V}$. 
-An atom from a surface or an interface is pushed into the bulk of the crystal (I). The required Gibbs potential is called $\Delta G_{I}$.

-A Frenkel defect is created by pushing a lattice atom into an I site. The Gibbs potential for this process is $\Delta G_{F}$.

From thermodynamics the formulae for the equilibrium concentrations of point defects can be derived. $C_{S}$ is the concentration of silicon atoms in the crystal.

$$
C_{V}^{e q}=C_{S} \cdot e^{-\Delta G_{V} / k T} \quad C_{I}^{e q}=C_{S} \cdot e^{-\Delta G_{I} / k T} \quad C_{F}=C_{S} \cdot e^{-\Delta G_{F} / 2 k T}
$$

The surface of a crystal can be active or passive. Passive means that the point defects cannot react with the surface, they can only be produced in the bulk by the Frenkel pair mechanism. Many experiments show that a silicon nitride surface is nearly passive $[6,8]$. An active surface reacts with the point defects generating and annihilating each kind of point defects independently. Silicon oxide surfaces are known to be active. For an infinite crystal or a crystal with a passivated surface the Frenkel mechanism is the only source of point defects. In this case V and I can only be generated and annihilated as pairs and $C_{F}$ is the equilibrium concentration value for $\mathrm{V}$ and $\mathrm{I}$. However, if the surface is activated, the resulting equilibrium values will change according to the above formulas for $C_{V}^{e q}$ and $C_{I}^{e q}$. The following considerations will help to clarify this.

It is possible to define three Gibbs potentials.

$G_{S}$ - The surface or interface potential is defined by the process of bringing an atom from infinity outside the crystal to the crystal surface or interface.

$G_{I}$ - The I potential is defined by bringing an atom from infinity outside the crystal to an I site inside the crystal.

$G_{V}$ - The $\mathrm{V}$ potential is defined by bringing an atom from infinity outside the crystal into a vacancy site.

From the law of energy conservation the following equations are obtained:

$$
\Delta G_{V}=G_{S}-G_{V} \quad \Delta G_{I}=G_{I}-G_{S} \quad \Delta G_{F}=G_{I}-G_{V}
$$

There cannot be any difference between bringing an atom from a lattice site to the surface and then to an I site and bringing this atom directly from his lattice site to an I site. Hence

$$
\Delta G_{F}=\Delta G_{I}+\Delta G_{V}
$$

Equ.3 is inserted into Equ.1:

$$
C_{V}^{e q} \cdot C_{I}^{e q}=C_{F}^{2}
$$

This simple relation holds for equilibri im point defect concentrations. In thermal equilibrium the generation of point defects by the Frenl:el pair mechanism and the generation by the surface mechanism are in a detailed balance. The Gibbs potentials $G_{I}$ and $G_{V}$ are independent of the position in a crystal. Under process conditions stress in the crystal could change its values. However, we do not consider any such change. We only take into account the change of the thermal equilibrium by the surface potential. This potential does not only depend on the bulk properties of the crystal, it also depends on the environment and on any kind of chemical reaction at the surface of the 
crystal. We conclude that any change of the surface potential will change the thermal equilibrium concentration of $\mathrm{V}$ and I inversely.

$$
C_{I}^{e q}=C_{S} \cdot e^{-\left(G_{I}-G_{S}\right) / k T} \quad C_{V}^{e q}=C_{S} \cdot e^{-\left(G_{S}-G_{V}\right) / k T}
$$

We denote by $C_{I}^{i n}$ and $C_{V}^{i n}$ the thermal equilibrium concentrations for an inert ambient. Both concentrations can be evaluated from the thermodynamic potentials:

$$
C_{I}^{i n}=C_{S} \cdot e^{-\left(G_{I}-G_{S}^{i n}\right) / k T} \quad C_{V}^{i n}=C_{S} \cdot e^{-\left(G_{S}^{i n}-G_{V}\right) / k T}
$$

By a suitable treatment of the surface of the crystal(e.g. by a chemical reaction) it is possible to change the surface potential.

$$
C_{I}^{e q}=C_{I}^{i n} \cdot e^{\Delta G_{S} / k T} \quad C_{V}^{e q}=C_{V}^{i n} \cdot e^{-\Delta G_{S} / k T} \quad \Delta G_{S}=G_{S}-G_{S}^{i n}
$$

These formulas show that a chemical reaction at the surface can produce $\mathrm{I}$ and annihilate $\mathrm{V}$ at the same time. From many experiments it is obvious that I are produced during thermal oxidation. But most of the oxidation models use a generation term of I and constant values of the thermal equilibrium concentrations of point defects. This would keep the surface: $\mathrm{V}$ concentration at a higher value compared with the case of pure bulk recombination. Therefore the ORD would not occur near the surface and would have a very strong dependence on the depth. Some models use an annihilation term for $\mathrm{V}$ to prevent this effect. In our model a bulk recombination effect is not needed because the undersaturation of $\mathrm{V}$ is produced directly at the surface. The difference between this model and the others is the variability of the thermal equilibrium concentrations of point defects. The Gibbs surface potential is changing in time and the concentration of point defects will follow these changes. The above considerations lead to general expressions for the boundary conditions for $\mathrm{V}$ and I. First of all we consider a highly active surface:

$$
C_{I}=C_{I}^{e q}(t) \quad C_{V}=C_{F}^{2} / C_{I}^{e q}(t)
$$

The concentration of point defects follows immediately the equilibrium concentration. For a lower surface activity the introduction of a reaction velocity is necessary:

$$
J_{I}=-k_{I}\left(C_{I}-C_{I}^{e q}(t)\right) \quad J_{V}=-k_{V}\left(C_{V}-C_{F}^{2} / C_{I}^{e q}(t)\right)
$$

The concentrations of point defects will follow the equilibrium concentration with a certain delay of time.

3. The variation of the Gibbs potential at the $\mathrm{Si}-\mathrm{SiO}_{2}$ interface under oxidizing conditions.

We consider a $\mathrm{SiO}_{2}$ layer covering a 1.onocrystalline silicon sample in an inert ambient. Under thermal equilibrium conditions there i : an exchange of silicon atoms between the $S i$ crystal and the $\mathrm{SiO}_{2}$ layer. The $\mathrm{Si}$ atoms have a certain partial pressure $\mathrm{P}$ at the $\mathrm{Si}-\mathrm{SiO}_{2}$ interface. From van't Hoff's law it is known that a solution with small density behaves like an ideal gas. The Gibbs potential per atom is equal to the chemical potential and can be expressed for the inert case by:

$$
G_{S}^{i n}=G_{0}+k T \ln \left(P_{i n}\right)
$$


Under oxidizing conditions the partial pressure at the interface will change:

$$
G_{S}^{o x}=G_{0}+k T \ln \left(P_{o x}\right)
$$

$G_{0}$ is a constant and independent of pressure. For the description of the variation of the Gibbs potential $G_{0}$ is not important.

$$
\Delta G_{S}=G_{S}^{o x}-G_{S}^{i n}=k T \ln \left(P_{o x} / P_{i n}\right)
$$

Therefore the variation of the chemical potential depends only on the partial pressure of silicon at the interface. Thus the formulas for the equilibrium concentration of $\mathrm{I}$ and $\mathrm{V}$ can be written in a simple form:

$$
C_{I}^{e q}=C_{I}^{i n} \cdot P_{o x} / P_{i n} \quad C_{V}^{e q}=C_{V}^{i n} \cdot P_{i n} / P_{o x}
$$

The above considerations reduce the problem of determination of the I concentration to the calculation of the relation between the values of partial pressure of $S i$ atoms in the oxide under oxidizing and inert conditions. In general the partial pressure is higher in oxidizing than in inert atmosphere. But under oxidizing conditions the oxygen is also dissolved in the oxide and tends to reduce the pressure of $S i$ by reacting with the $S i$ atoms. Therefore a source of $S i$ must exist in the silicon oxide layer. This source can be found in the following chemical reaction:

$$
2 \mathrm{SiO} \rightleftharpoons \mathrm{Si}+\mathrm{SiO}_{2}
$$

The silicon is not completely oxidized to $\mathrm{SiO}_{2}$, because a part of the $\mathrm{Si}$ atoms is only oxidized to $\mathrm{SiO}$. Under the assumption that this reaction occurs near the $\mathrm{Si}-\mathrm{SiO}_{2}$ interface, it is possible to express this silicon atom source as a current which is proportional to the oxide growth rate.

$$
J_{\text {Source }}=f v_{o x}
$$

$f$ is the fraction of silicon atoms, which are oxidized to siliconmonoxide. $J_{S o u r c e}$ is the current of produced silicon atoms. However we cannot directly use this current as a boundary condition because a part of the atoms fills up the new growing oxide layer and does not flow into the already formed silicondioxide. Therefore a correction term has to be included:

$$
J_{S i}=f v_{o x}-C_{S i} v_{o x}
$$

$C_{S i}$ is the concentration of $S i$ atoms at the interface and $C_{S i} v_{o x}$ is the amount of silicon that flows into the new oxide per unit of time. J'he silicon atoms diffuse into the $\mathrm{SiO}_{2}$ and react with the oxygen. Under the assumption that the oxygen partial pressure is constant in the reaction area and the diffusion of $S i$ atoms is faster shan the oxide growth rate, we obtain

$$
\frac{\partial C_{S i}}{\partial t}=D_{S i} \frac{\partial^{2} C_{S i}}{\partial x^{2}}-r C_{S i} C_{O_{2}}
$$

$r$ is a reaction constant. From the model of Deal and Grove it is known that the oxygen partial pressure is proportional to the oxide growth rate. Thus under thermal equilibrium $\left(\partial C_{S i} / \partial t=0\right)$ the equation becomes:

$$
D_{S i} \frac{\partial^{2} C_{S i}}{\partial x^{2}}=k v_{o x} C_{S i}
$$


Let us denote by $x=0$ the $S i-S i O_{2}$ interface and by $x=d$ the $S i O_{2}$ surface in which $d$ is the oxide thickness. Then the analytical solution of eq.(18) with the boundary conditions $J_{S i}(d)=0$ and $J_{S i}(0)=J_{S i}$ as given by eq.(16) yields:

$$
C_{S i}=\frac{J_{S i}}{A D_{S i}\left(1-e^{-2 A d}\right)}\left(e^{-A x}+e^{A(x-2 d)}\right) \quad A=\sqrt{\frac{k v_{o x}}{D_{S i}}}
$$

Inserting $J_{S i}$ from eq.(16) we obtain the $S i$ atom concentration $C_{S i}=C_{S i}(0)$ at the $S i-S i O_{2}$ interface:

$$
C_{S i}=\frac{f}{1+\sqrt{k D_{S i} / v_{o x}} \cdot \tanh \left(d \sqrt{k v_{o x} / D_{S i}}\right)}
$$

and

$$
P_{o x} / P_{i n}=\frac{s u_{0}}{1+\sqrt{k D_{S i} / v_{o x}} \cdot \tanh \left(d \sqrt{k v_{o x} / D_{S i}}\right)}
$$

$s u_{0}\left(=f / C_{S i}^{i n}\right)$ is the supersaturation for thin oxide layers $(d=0)$. The above formula combined with the formula of Deal and Grove yields a $t^{-1 / 4}$ law for the supersaturation as found in many OED experiments. For very slow oxidation rates ORD is observed instead of OED. The model correctly accounts also for this effect. Fig 1.[1] and 2.[2] show the good agreement for short and for long oxidation time, respectively. The Model of Massoud [9] determines the oxide growth rate.

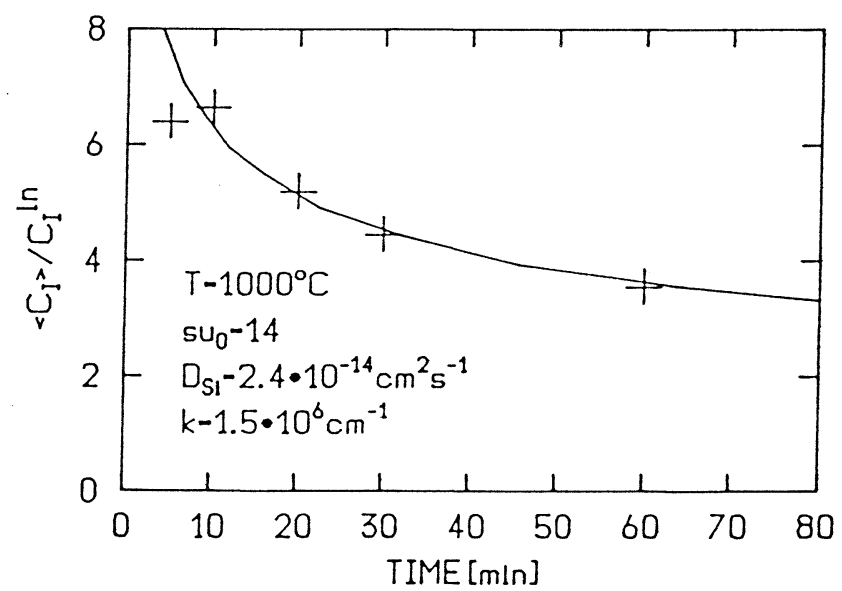

Fig.1

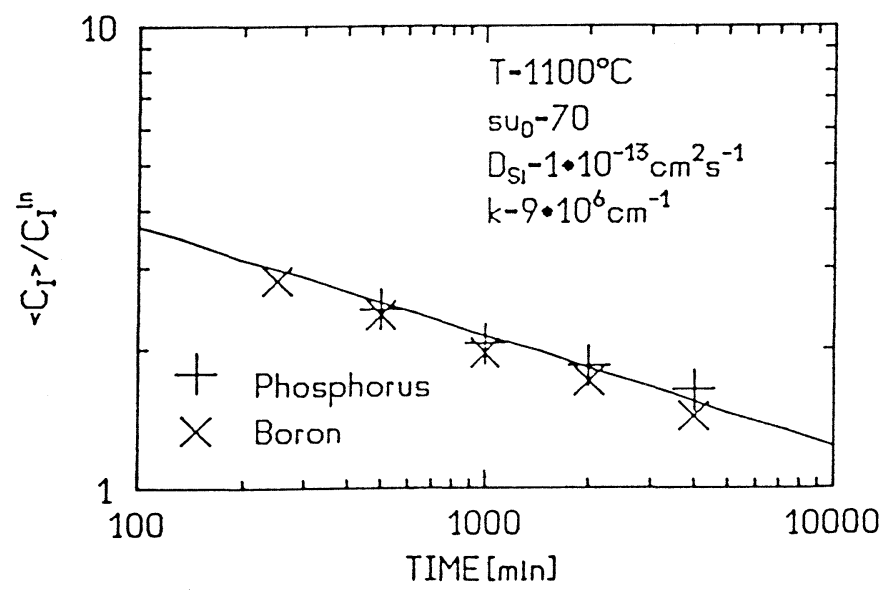

Fig.2

4. Determination of point defect equilibrium concentrations in silicon

In order to determine the absolute values of the equilibrium point defect concentrations we use the general purpose one dimensional process simulator ZOMBIE [4] which solves simultaneously the diffusion equations for $\mathrm{V}$ and $\mathrm{I}$ uncer appropriate boundary conditions and yields the average concentration of $\mathrm{V}$ and $\mathrm{I}$ respectively. Under silicon nitride layers and inert conditions only the Frenkel pair mechanism can produce point defects. Therefore the equilibrium concentration for $\mathrm{V}$ and I must be equal. A comparison b tween diffusion of dopants under nitride and under oxide interfaces indicates that the equilibriun . concentration of both point defects must also be nearly the same for oxide interfaces $[6,7]$. In our calculations the products of point defect diffusion coefficient and equilibrium concentration for botlı kinds of point defects were taken from the results of the gold diffusion experiments by Morehead et al [5]. The absolute values of equilibrium point defect concentrations were now obtained by comparison of our calculations with the experiments of Mizuo and Higuchi [8] in which the average I concentration is given by the OED of phosphorus. Under 
the assumption of $C_{I}^{i n}=C_{V}^{i n}=1.4 \cdot 10^{16} \mathrm{~cm}^{-3}, D_{I}=1.1 \cdot 10^{-7} \mathrm{~cm}^{2} \mathrm{~s}^{-1}$ and $D_{V}=2.9 \cdot 10^{-9} \mathrm{~cm}^{2} \mathrm{~s}^{-1}$ for $1100^{\circ} \mathrm{C}$ we obtain a fair agreement between the results of our calculations and the experiments as shown in Fig.3 to Fig.4. The fairly good agreement supports our model.

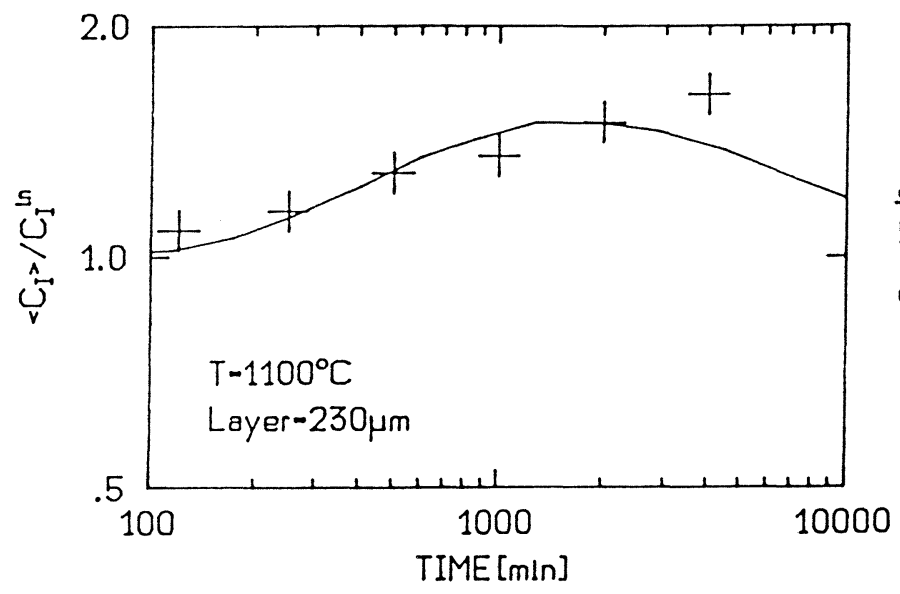

Fig.3

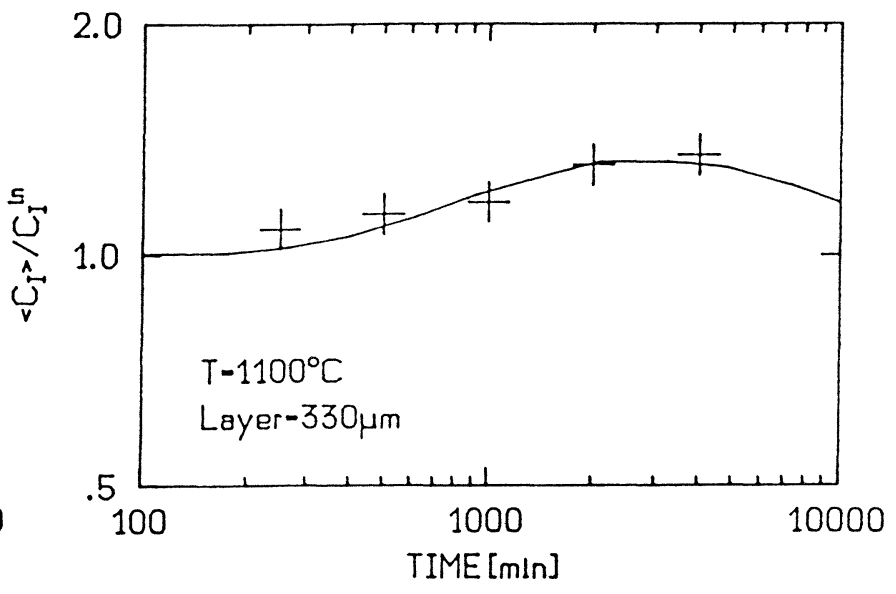

Fig.4

5. Summary and conclusions

This work presents a general law for boundary conditions of point defect distributions. The equilibrium concentrations of point defects is determined by the condition at the surface. This condition only can change the equilibrium concentrations inversely and is always in a detailed balance with the bulk. Additionally a model for the condition at the surface during thermal oxidation is presented that explains the observed phenomena and is useful to simulate the experiments of Mizuo and Higuchi. The simulation yields the values of the equilibrium concentrations of both kinds of point defects at $1100^{\circ} \mathrm{C}$.

\section{Acknowledgement}

The authors thank Prof. Goesele for helpful encouragement. This work has been supported by the Fonds zur Foerderung der Wissenschaftlichen Forschung Projekt No. S43/10 and by the Siemens Research Laboratories, Munich, W. Germany. Thanks are also due to our Computer Center for free computer access.

\section{References}

[1]D.A.Antoniadis,I.Moskowitz:J.Appl.Phys.,Vol.53,pp.6788(1982)

[2]T.Y.Tan,U.Goesele.Appl.Phys.Lett.,Vol.40,pp.616(1982)

[3]A.M.Lin,R.W.Dutton,D.A.Antoniadis, W.A.Tiller:J.Electrochem.Soc.,Vol.128 pp.1121(1981)

[4]W.Juengling,P.Pichler,S.Selberherr,J'.Guerrero,H.Poetzl:IEEE Trans.ED-32, pp.156(1985)

[5]F.Morehead et al:Appl.Phys.,Vol.59. pp.690(1983)

[6]S.Mizuo,H.Higuchi:Jpn.J.Appl.Phys ,Vol.21,pp.281(1982)

[7]S.Mizuo,H.Higuchi:Jpn.J.Appl.Phys ,Vol.21,pp.56(1982)

[8]S.Mizuo,H.Higuchi:J.Electrochem.Soc.,Vol.129,pp.2292(1982)

[9]H.Z.Massoud,C.P.Ho,J.D.Plummer,Final Report for Period 15Feb81-31Mar82, Integrated Circuit Lab.,Standford University,CA94305,pp.194,(1982) 